\title{
VP22 herpes simplex virus protein can transduce proteins into stem cells
}

\author{
I. Gabanyi ${ }^{1}$, F.H. Lojudice ${ }^{1}$, P.M. Kossugue ${ }^{1}$, E. Rebelato ${ }^{2}$, M.A. Demasi ${ }^{1}$ and M.C. Sogayar ${ }^{1}$ \\ ${ }^{1}$ Centro de Terapia Celular e Molecular, Departamento de Bioquímica, Instituto de Química, Universidade de São Paulo, \\ São Paulo, SP, Brasil \\ ${ }^{2}$ Departamento de Fisiologia e Biofísica, Instituto de Ciências Biomédicas, Universidade de São Paulo, São Paulo, SP, Brasil
}

\begin{abstract}
The type I herpes simplex virus VP22 tegument protein is abundant and well known for its ability to translocate proteins from one cell to the other. In spite of some reports questioning its ability to translocate proteins by attributing the results observed to fixation artifacts or simple attachment to the cell membrane, VP22 has been used to deliver several proteins into different cell types, triggering the expected cell response. However, the question of the ability of VP22 to enter stem cells has not been addressed. We investigated whether VP22 could be used as a tool to be applied in stem cell research and differentiation due to its capacity to internalize other proteins without altering the cell genome. We generated a VP22.eGFP construct to evaluate whether VP22 could be internalized and carry another protein with it into two different types of stem cells, namely adult human dental pulp stem cells and mouse embryonic stem cells. We generated a VP22.eGFP fusion protein and demonstrated that, in fact, it enters stem cells. Therefore, this system may be used as a tool to deliver various proteins into stem cells, allowing stem cell research, differentiation and the generation of induced pluripotent stem cells in the absence of genome alterations.
\end{abstract}

Key words: VP22 protein delivery; Protein transducing domain; eGFP; Stem cells transduction; Cell differentiation

\section{Introduction}

Cell transplantation is a valid approach to treat some types of diseases; however, this treatment is limited by the low availability of many types of cells.

Stem cell differentiation is a valuable tool to approach this problem, but several issues have to be overcome before it can be used on a regular basis for cell therapy. Safety is an important issue, especially when cell differentiation is achieved by stable gene expression, due to the risk of mutagenesis, which may be associated with prolonged expression of the transgene (1).

An attractive alternative is proteins containing a protein transduction domain (PTD), which are characterized by their ability to cross the plasma membrane and translocate into living cells. The most commonly studied PTD proteins are: the human immunodeficiency virus (HIV-1) TAT protein, the Drosophila Antennapedia transcription factor and the herpes simplex virus type 1 (HSV1) VP22 protein (2).

PTD proteins contain a short arginine- or lysine-rich peptide sequence. These basic amino acids seem to be important for the contact with the negatively charged lipids present in the cell membrane, which is the first step of protein transduction (3). Several theories have been proposed to explain how these PTD proteins enter the cell $(4,5)$, but the actual mechanism is yet to be understood.

These PTD proteins have been used as tools to deliver a variety of other proteins into different cell types, leading to the expected biological response $(6,7)$. Protein transduction in vivo has also been reported $(3,8,9)$. The capacity of PTD proteins to deliver a variety of other cargos, such as RNAi, siRNA, iron beads, liposomes, and plasmids has also been reported (10).

VP22, a 301-amino acid protein encoded by the UL49 gene, is found in the HSV-1 tegument, being highly phosphorylated and carrying an arginine-rich PTD in its Cterminal. VP22 is one of the most abundant proteins of the tegument, with approximately 2000 copies per virion. VP22 is packaged into the virion during the final stages of envelopment, but its role in viral infection is still not well understood. In addition to important features such as microtubule binding, nuclear translocation during mitosis, chromatin and nuclear membrane binding, VP22 also displays capacity for intercellular trafficking $(11,12)$. 
Although the intercellular trafficking capacity of VP22 has been shown for many cell types both in vitro $(6,13-15)$ and in vivo $(8,9)$, some investigators have claimed that this could be attributed to fixation artifacts $(4,16)$. Moreover, no VP22 transduction studies have been addressed towards stem cells.

Since stem cells usually behave differently than most cells regarding transfection and heterologous gene expression (17), we set out to evaluate the ability of VP22 to translocate into stem cells and also to deliver other proteins to these cells in the absence of permanent genome alteration

To this end, we generated a VP22.eGFP (enhanced green fluorescent protein) construct to be used as a tool to evaluate the ability of VP22 to translocate and deliver other proteins into two types of stem cells, namely mouse embryonic stem cells (mESCs) and human dental pulp stem cells (hDPSCs).

\section{Material and Methods}

\section{Vector construction}

The open reading frame of the eGFP protein was obtained by digesting the pNF $\mathrm{B}-\mathrm{d} 2 \mathrm{EGFP}$ (Clontech, USA) vector with the HindIII restriction enzyme (Fermentas, USA). The fragment obtained was then sub-cloned into the pLPCX vector (Clontech), which had been previously digested with the same enzyme, using T4 DNA ligase (Fermentas) according to manufacturer instructions. The same process was used to sub-clone the eGFP into the pVP22 vector (Invitrogen, USA). These vectors were used separately to transform Escherichia coli bacteria (DH10B strain) through electroporation, leading to bacterial clones carrying the recombinant pLPCX.eGFP or pVP22.eGFP vectors. Correct DNA sequence and frame were confirmed by DNA sequencing.

\section{hDPSC culture conditions}

hDPSCs were obtained from normal human extracted third molars for which the donors gave informed consent. Tooth surfaces were cleaned to eliminate other tissue around the teeth. The pulp was digested in a solution of $3 \mathrm{mg} / \mathrm{mL}$ type IA collagenase (Sigma-Aldrich, Brazil) and $4 \mathrm{mg} / \mathrm{mL}$ dispase (Roche, Brazil) for $1 \mathrm{~h}$ at $37^{\circ} \mathrm{C}$. Singlecell suspensions were seeded onto plastic flasks with alpha modified Eagle's medium ( $\alpha$-MEM; Sigma-Aldrich) supplemented with 10\% FCS (Cultilab, Brazil), and ciprofloxacin (Bayer, Brazil) and incubated at $37^{\circ} \mathrm{C}$ in $5 \% \mathrm{CO}_{2}$. These cells were characterized as mesenchymal stem cells according to their surface membrane markers (18), being negative for CD14, CD34, CD45 hematopoietic and CD31 endothelial markers and positive for CD29, CD44 and CD90 mesenchymal markers, and also due to their differentiation potential into adipocytes and osteoblasts (Kossugue PM, Lojudice FH, Sogayar $\mathrm{MC}$, unpublished results).

\section{mESC culture conditions}

mESCs from the USP4 lineage (kindly provided by Dr. Lygia da Veiga Pereira, Bioscience Institute, University of São Paulo, Brazil) were maintained over a layer of murine-inactivated fibroblasts with Dulbecco's modified Eagle's medium (DMEM; Sigma-Aldrich) supplemented with $15 \%$ FCS-ES certified for stem cell cultivation (Hyclone, USA), 2 mM L-glutamine (Ajinomoto, Brazil), $1 \times$ MEM-non-essential amino acids (Gibco, USA), $1 \times$ $10^{3} \mathrm{U} / \mathrm{mL}$ murine leukemia inhibitory factor (Chemicon, USA), $0.1 \mathrm{mM} \beta$-mercaptoethanol (Gibco), $10 \mu \mathrm{g} / \mathrm{mL}$ ciprofloxacin (Bayer), and incubated at $37^{\circ} \mathrm{C}$ in $5 \% \mathrm{CO}_{2}$. The complete characterization of these cells has been described in Ref. 19

\section{T and Chinese hamster ovary ( $\mathrm{CHO}$ ) cell culture conditions}

293T and $\mathrm{CHO}$ cells were maintained in DMEM supplemented with $10 \% \mathrm{FCS}$ and $10 \mu \mathrm{g} / \mathrm{mL}$ ciprofloxacin and incubated at $37^{\circ} \mathrm{C}$ in $5 \% \mathrm{CO}_{2}$.

\section{Transient and stable transfection}

293T and $\mathrm{CHO}$ cells were transfected with the desired vector (pVP22, pVP22.eGFP or pLPCX.eGFP) using Lipofectamine 2000 (Invitrogen) according to manufacturer instructions, using $10^{6}$ cells $/ 35-\mathrm{mm}$ plate and $4 \mu \mathrm{g}$ of the vector preparation. Protein extracts or conditioned culture medium from 293T cells were obtained within 48$72 \mathrm{~h}$ after transfection. CHO cells were transfected and, after $48-72 \mathrm{~h}$, the cultures were replated at low density and subjected to selection in the presence of Geneticin G418 (800 $\mu \mathrm{g} / \mathrm{mL}$; Invitrogen) in order to select for stable cell clones expressing the VP22 protein or the VP22.eGFP fusion protein.

\section{Western blot analysis}

Cells were harvested into RIPA ${ }^{+}$lysis buffer (10 mM Tris- $\mathrm{HCl}, \mathrm{pH} 7.5,1 \%$ sodium deoxycholate, $1 \% \mathrm{NP}-40$, $150 \mathrm{mM} \mathrm{NaCl}, 0.1 \%$ SDS, $1 \mathrm{mM}$ DTT and $1 \times$ protease inhibitors cocktail; GE Healthcare, USA). Protein samples $(50 \mu \mathrm{g})$ of total protein or $30 \mu \mathrm{L}$ of culture medium were fractioned by SDS-PAGE. Gels were blotted onto a nitrocellulose membrane (Bio-Rad, USA), which was blocked with 5\% non-fat milk in Tris-buffered saline (TBS) containing $0.05 \%$ Tween 20 , overnight at $4{ }^{\circ} \mathrm{C}$ After 3 washes with TBS $/ 0.05 \%$ Tween 20 , the membranes were incubated with polyclonal antiserum to VP22 (1:800 dilution; kindly provided by Dr. Stuart Perkins, Biomedical Sciences Department, Wiltshire, UK) diluted in the same buffer containing $5 \%$ non-fat milk for $1 \mathrm{~h}$ at room temperature. The membranes were washed again and then probed with horseradish peroxidase-conjugated secondary antibodies (Vector Laboratories, USA). The signals were detected using the ECL-Plus detection system (GE Healthcare) according to manufacturer instructions. 


\section{VP22.eGFP transduction assay}

mESCs were subjected to embryoid body formation for 5 days and adhesion for 3 days prior to the VP22.eGFP transduction assay. hDPSCs or mESCs $\left(\sim 2 \times 10^{5}\right.$ cells) were seeded onto $60-\mathrm{mm}$ plates containing two coverslips, each treated with $150 \mu \mathrm{L}$ of the 293T cell extracts mixed with $150 \mu \mathrm{L}$ of KrebsHanseleit solution without $\mathrm{NaCl}$ and supplemented with $11.2 \mathrm{mM}$ glucose for $30 \mathrm{~min}$. Cells were then washed with Krebs-Hanseleit solution and incubated with DAPI (10 ng/ $\mathrm{mL}$; Sigma) in Krebs-Hanseleit solution for $10 \mathrm{~min}$, washed again and observed by confocal microscopy.

The 293T cell protein extracts were obtained $48 \mathrm{~h}$ after transfection of $6 \times 10^{6} 293 \mathrm{~T}$ cells with $24 \mu \mathrm{g}$ of either pVP22.eGFP or pLPCX.eGFP vectors, using icecold high salt buffer (10 mM HEPES, pH 7.9; 400 mM $\mathrm{NaCl}$; $0.1 \mathrm{mM}$ EDTA; $0.5 \mathrm{mM}$ DTT; $5 \%$ glycerol) according to the manufacturer protocol (Invitrogen). These extracts were obtained simultaneously on the day of treatment. To ensure reproducibility of the treatment, each experiment was repeated three times, using four coverslips each time, namely two treated with eGFP and two with VP22.eGFP.

\section{Flow cytometry analysis}

Cell extracts were obtained from 293T cells transfected with either the pVP22.eGFP or the pLPCX.eGFP vector, as described earlier. These extracts were added to the culture medium of hDPSC cultures and $24 \mathrm{~h}$ later these cells were harvested with trypsin, washed twice with PBSA and subjected to flow cytometry. All analyses were carried out using a FACS-Calibur ${ }^{\circledR}$ flow cytometer and the CELLQuest ${ }^{\circledR}$ analysis program (Becton Dickinson, USA). eGFP was detected at $488 \mathrm{~nm}$ by laser scanning. The results were normalized relative to the auto-fluorescence of the untreated cells.

\section{Confocal microscopy analysis}

Fluorescence of the 293T cells transiently transfected with the pVP22.eGFP construct and the stem cells (hDPSCs and mESCs) transduced with VP22.eGFP were analyzed by confocal microscopy (Carl Zeiss Inc., USA). Prior to the confocal microscopy analysis, the cells were incubated with DAPI (10 ng/mL; Sigma) for $10 \mathrm{~min}$ and washed. The live/unfixed cells were visualized in an openperfusion chamber employing a confocal laser scanning microscope using two lasers with excitation wavelengths at $488 \mathrm{~nm}$ for eGFP and $350 \mathrm{~nm}$ for DAPI and anoil immersion lens $(63 \times)$. For mESCs and 293T, the fluorescence was captured from whole cells. Image stacks of confocal optical sections $(\sim 0.5 \mu \mathrm{m})$ of the hDPSCs were acquired in z-stacks. Images were analyzed using the manufacturer software.

\section{Real-time quantitative RT-PCR (qRT-PCR)}

hDPSCs were co-cultured using the Millicell cell culture insert with the Hanging Geometry system (Millipore, USA) for 6 days along with $\mathrm{CHO}$ cells expressing VP22 or VP22.eGFP, using parental $\mathrm{CHO}$ cells as control. After this period, total RNA was isolated from hDPSCs using RNeasy mini kits (Qiagen, Germany) and cDNA was synthesized from $1 \mu \mathrm{g}$ RNA using Superscript II (Invitrogen). qRT-PCR was performed using gene-specific primers with Platinum SYBR Green qPCR Supermix (Applied Biosystems, USA) according to manufacturer instructions and the results were analyzed with the 7300 System Software V1.3.1. The primers were designed using the Primer Express program and the betatubulin gene transcript levels were used as the internal control. The experiments were carried out in triplicate.

\section{Results}

\section{Generation of the VP22.eGFP construct and confirmation of fusion protein expression}

In order to test the ability of VP22 to enter cultured stem cells, we generated an expression vector containing VP22 coupled to eGFP (pVP22.eGFP; Figure 1A). To confirm its ability to yield the desired fusion protein (VP22.eGFP of $\sim 70 \mathrm{kDa}$ ), this construct was transfected into 293T cells, and these cells were subjected to analysis by both Western blotting (Figure 1B) and confocal microscopy (Figure $1 \mathrm{C}$ ), revealing the production of the desired fusion protein.

\section{Presence of the VP22 protein in the culture medium of the producing cells}

To document the capacity of VP22 to penetrate and cross membranes, we first analyzed whether the VP22 protein and the VP22.eGFP fusion protein were secreted by the cells and could be detected in the culture medium. We found that the VP22 protein and the newly constructed VP22.eGFP fusion protein were able to transpose the membrane of their overproducing cells and could be detected in the culture medium by Western blotting (Figure 1D).

\section{VP22 transduction into stem cells}

In order to investigate the ability of VP22 to transduce stem cells, we treated hDPSCs (Figure 2A and C) or mESC (Figure 2B) with protein extracts obtained from 293T cells transiently expressing the VP22.eGFP fusion protein. Knowing the controversy about the ability of VP22 to enter living cells, we decided to perform a confocal microscopy analysis to show in living cells that hDPSCs and mESC treated with VP22.eGFP had, in fact, incorporated the eGFP protein. Using a confocal z-stack scan, we were able to demonstrate that this protein was indeed in the interior of hDPSCs and not only attached to the membrane (Figure 2A; upper panels). As a negative control, we treated hDPSCs with cell extracts obtained from 293T cells transiently expressing eGFP alone, with 

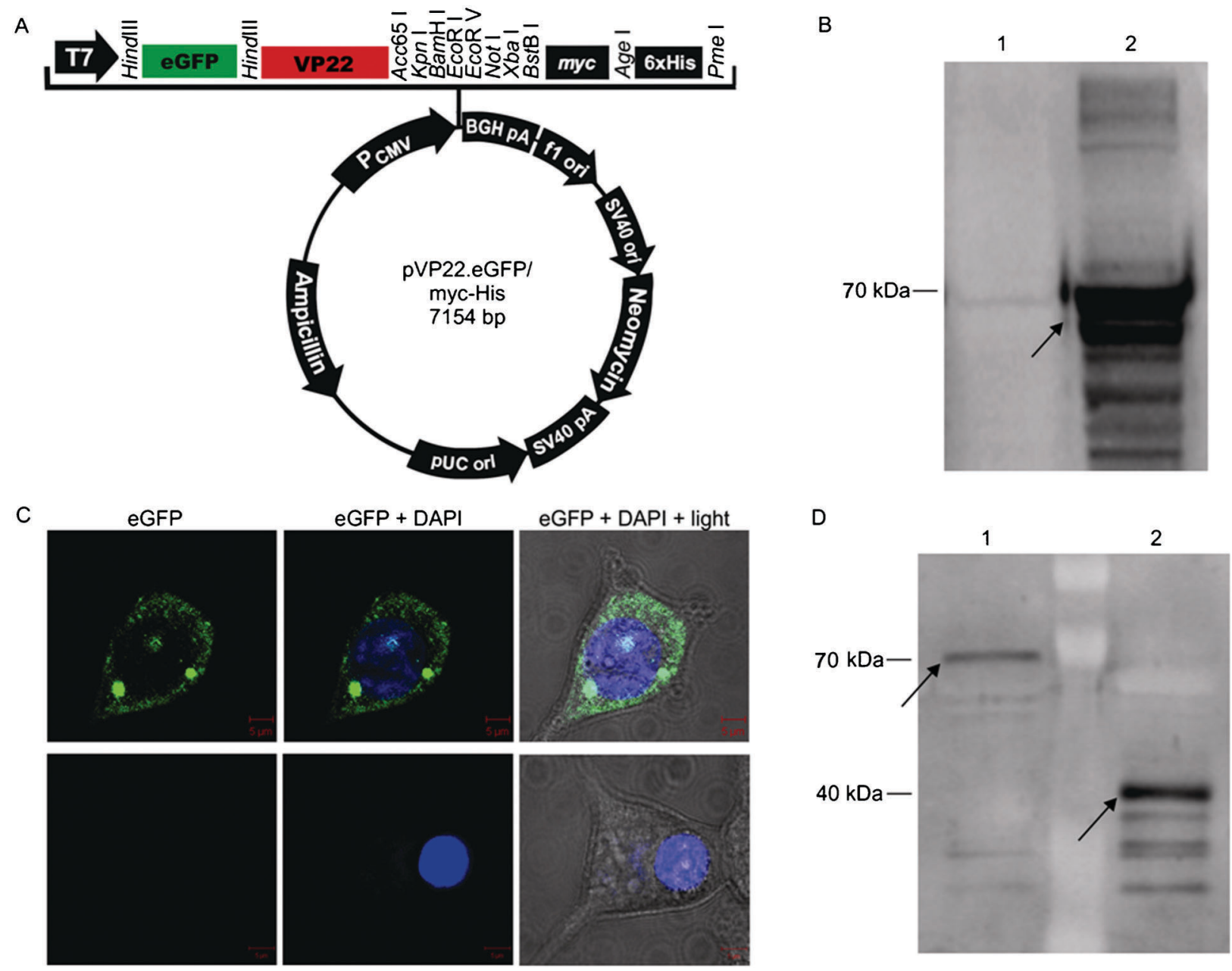

Figure 1. $A$, Schematic drawing of the vector constructed and used in the present study, pVP22.eGFP. $B$, The expression of the VP22.eGFP fusion protein was confirmed by Western blot using an anti-VP22 antibody. The 293T cells transfected with pLPCX.eGFP were used as a negative control (lane 1) and the extract obtained from 293T cells transfected with pVP22.eGFP (lane 2) showed a band of $\sim 70 \mathrm{kDa}$ corresponding to the fusion of eGFP $(\sim 30 \mathrm{kDa})$ to VP22 $(\sim 40 \mathrm{kDa})$; the arrow indicates the band. C, The 293T cells transfected with the pVP22.eGFP vector were analyzed with a confocal microscope. The upper panels show the cells with green fluorescence due to the presence of eGFP and the lower panels show the control cells that were not transfected. DAPI staining was used to define the cell nucleus. $D$, Detection of VP22 and VP22.eGFP proteins in the culture medium of the respectively producing cells. The culture medium of 293T cells transfected with either VP22.eGFP (lane 1) or VP22 (lane 2) was subjected to Western blot analysis, revealing the expected band corresponding to VP22 ( $40 \mathrm{kDa})$ and VP22.eGFP ( $70 \mathrm{kDa})$; arrows indicate these bands.

no fluorescence being found inside these cells (Figure 2A; lower panels).

To estimate the number of cells that incorporated the VP22.eGFP protein and the period of time during which these proteins could be shown inside the cells, we treated the hDPSCs with cell extracts obtained from 293T cells transiently expressing eGFP alone or VP22.eGFP. After $24 \mathrm{~h}$, we analyzed the eGFP protein present in these cells by flow cytometry. The results showed that $1.1 \%$ of the cells treated with the cellular extract containing eGFP were fluorescent, whereas, $10.3 \%$ of fluorescent cells were observed upon treatment with the cellular extract containing VP22.eGFP (Figure 2C).

For mESCs, we used wide field confocal microscopy, and observed eGFP fluorescence only in cells treated with the extract containing VP22.eGFP, while no fluorescence was found in cells treated with the extract containing only eGFP or in the control cells without any treatment (Figure 2C). A confocal z-stack scan was also performed on these cells in order to ensure that the VP22.eGFP fusion protein was inside the cell (data not shown). These results demonstrate that VP22 is capable of delivering an active protein, in this case eGFP, into both hDPSCs and mESCs. 

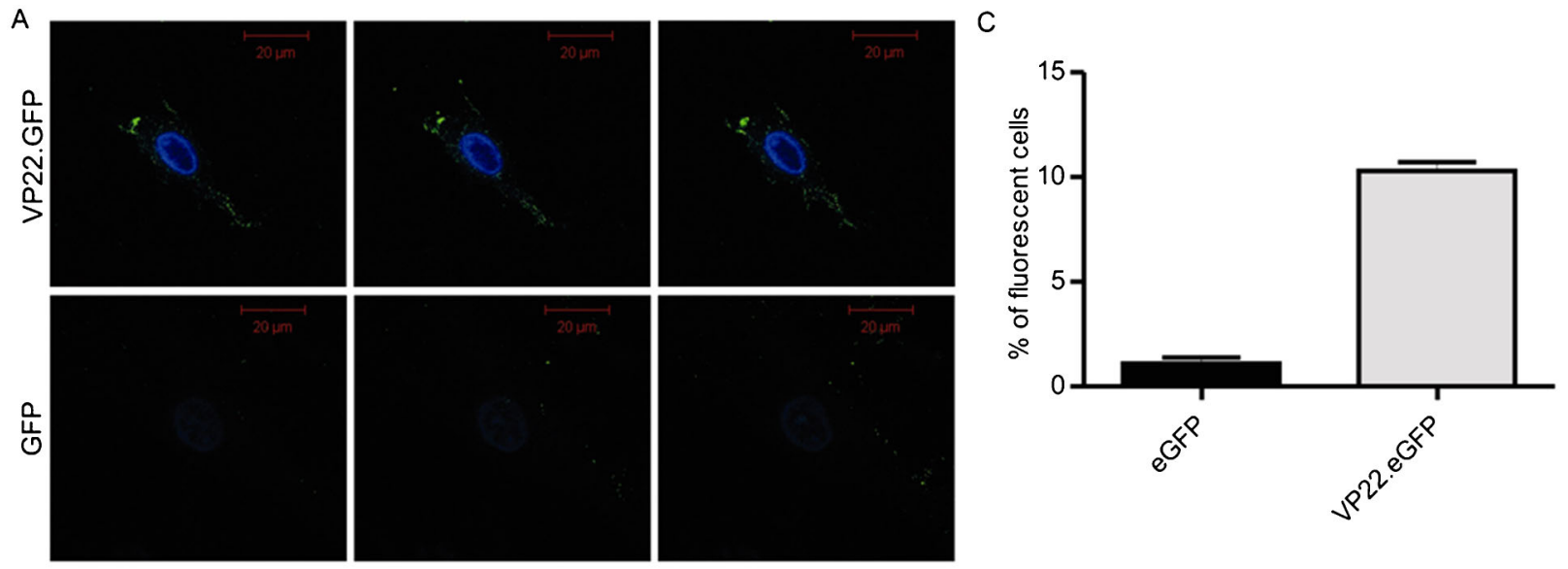

B

control

VP22
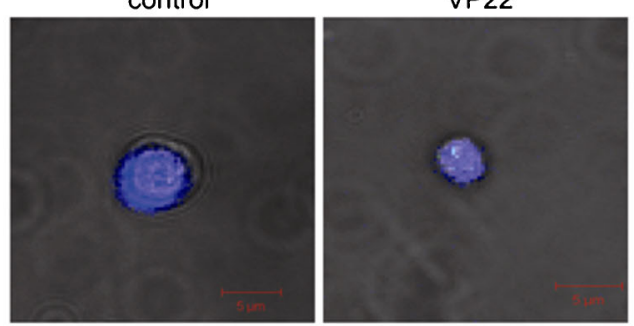

VP22.GFP

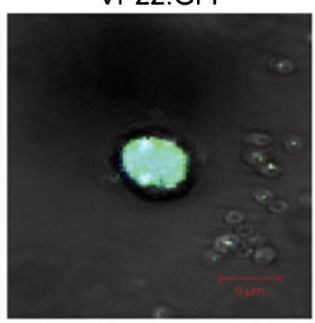

Figure 2. VP22.eGFP transduction into stem cells. A, hDPSCs showed green fluorescence after 30 min of incubation with protein extracts containing VP22.eGFP (upper panels); the negative control, incubated for the same period of time with the protein extracts obtained containing eGFP alone showed no fluorescence inside the cell (lower panels). hDPSCs were visualized by confocal microscopy, and optical slides of $\sim 0.5 \mu \mathrm{m}$ were obtained. The Figure shows a representative cell for each condition on three different optical slides. $B$, The mESCs were observed under a confocal microscope in a wide field. The cells treated with protein extracts containing VP22.eGFP showed green fluorescence; the cells incubated with the protein extracts obtained containing eGFP revealed no fluorescence. Also, mESCs with no treatment were visualized by confocal microscopy as a control for the possible auto-fluorescence of the cell (control). DAPI staining was used to define the nucleus of the cell. The Figure shows a representative cell for each condition in a wide field. $C$, Flow cytometry analysis shows the quantitation of hDPSCs that incorporated eGFP after 24-h incubation with protein extracts obtained from 293T cells after $48 \mathrm{~h}$ of transfection with eGFP- or VP22.eGFP-expressing vectors.

OCT4

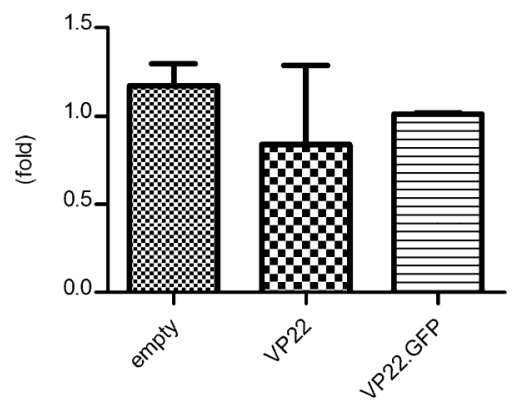

Nanog

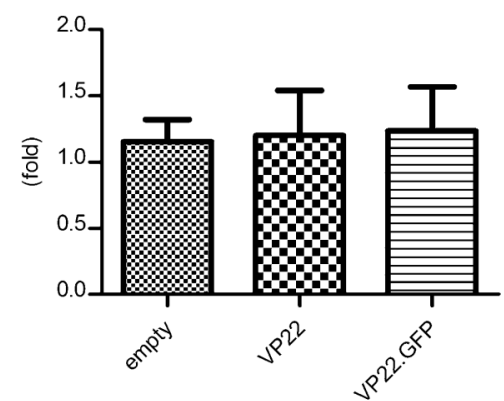

TRA1

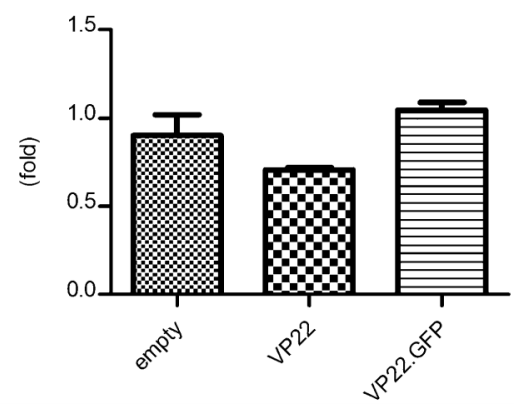

Figure 3. Detection of the mRNA expression level corresponding to the OCT4, Nanog, and TRA1 genes in hDPSCs by qRT-PCR. These cells were subjected to co-culture with $\mathrm{CHO}$ cells expressing VP22 or VP22.eGFP and with regular CHO cells (empty) as a control for 6 days prior to analysis. 


\section{Maintenance of the stem cell phenotype}

Since stem cells are sensitive to exogenous stimuli, we decided to investigate whether the VP22 or the VP22.eGFP fusion proteins could affect the phenotype of hDPSCs. To this end, we co-cultured the hDPSCs with CHO cells stably expressing VP22 or VP22.eGFP proteins for 6 days. We showed that none of the stem cell markers analyzed were affected by VP22 alone or by the VP22.eGFP fusion protein (Figure 3). Thus, no significant difference was found in the expression of OCT4, Nanog and TRA1 stem cell markers when compared to hDPSCs co-cultured with parental $\mathrm{CHO}$ cells or to the ones co-cultured either with $\mathrm{CHO}$ cells expressing VP22 or VP22.eGFP. These results also indicate that co-culturing of hDPSCs with $\mathrm{CHO}$ cells does not affect the stem cell phenotype of the former.

\section{Discussion}

Despite the controversy concerning the VP22 transduction capacity $(4,16)$, we have demonstrated for the first time in living stem cells the ability of VP22 to enter and deliver other proteins into two different types of stem cells, namely hDPSC and $\mathrm{mESC}$, proving the viability of employing VP22 as a tool in stem cell research and differentiation.

Some of the arguments against the VP22 transduction ability are that the fluorescence observed inside the cell was only visible after fixation. According to this argument, the fixation procedure would open some channels in the membrane and allow the VP22.eGFP protein attached to the membrane to enter the cell and migrate to the nucleus due to its positive charge (16).

To avoid any fixation artifacts, we developed a protocol to test the capacity of VP22 to enter stem cells using live/unfixed stem cells, and were able to demonstrate that VP22 is, in fact, capable of entering these cells and not simply adsorbing to the membrane. Moreover, we undertook confocal microscopy to probe the cells through different optical sections in order to demonstrate that the VP22.eGFP fusion protein was indeed inside the cells and not only adsorbed to the cell membrane, a situation that could not be distinguished using other techniques such as flow cytometry. The confocal microscopy results confirm that VP22 is, in fact, capable of transducing stem cells (Figure 2).

Here we show that the VP22.eGFP fusion protein is expressed by the producing cells and exported to the culture medium (Figure 1), being capable of entering the stem cells. Although we showed fluorescence only in the cytoplasm of the receptor cells (Figure 2A) we were able to visualize the eGFP fluorescence also in the nucleus of some other receptor cells (data not shown). It is also known that the VP22 present in the cytoplasm of receptor cells is able to reach the nucleus when these cells undergo mitosis $(11,12)$, allowing any protein fused to VP22 to exert its function in the nucleus of the cell, initiating a signaling cascade without any genome alteration.

Having established that, we used flow cytometry analysis to estimate the efficacy and durability of the ectopic protein expression using this method. We found that even after $24 \mathrm{~h}$ of treatment with the cellular extract containing VP22.eGFP we could observe fluorescence in $10.3 \%$ of the cells analyzed (Figure $2 \mathrm{C}$ ). Regarding the method used, we believe that a reasonable percentage of fluorescent cells was achieved, showing that the VP22 protein may be considered an efficient tool to deliver exogenous proteins into stem cells. We also believe that this efficacy may be increased when the cells are treated with the purified protein.

In the present study, we used a protein transduction protocol to demonstrate that the VP22 protein may be used as a tool to transduce stem cells without altering their genome. We adopted cellular extracts based on simplicity; however, we believe that, in order to deliver a protein aimed at changing the stem cell phenotype, the purified protein or co-culturing of the stem cells with cells producing the desired protein should be adopted.

In the co-culture method, the stem cells would be constantly receiving the protein fused to VP22. One issue is whether the VP22 protein may be considered an innocuous molecule when transduced into stem cells. To address this question, we co-cultured the hDPSCs with $\mathrm{CHO}$ cells expressing either VP22 or VP22.eGFP for 6 days and observed that after this period the cells were still expressing the OCT4, Nanog and TRA1 stem cell markers, showing that neither VP22 nor any other protein produced by the $\mathrm{CHO}$ cells seemed to interfere with the stem cell phenotype (Figure 3).

Several cell types have been used in VP22 studies, with satisfactory results $(6,8,9,13-15)$. However, to date, no reports have addressed the capacity of VP22 to enter any type of stem cell, which would make VP22 a valuable tool to be used in stem cell research and differentiation.

In a study seeking a possible treatment for Parkinson's disease, another type of PTD protein, the HIV virus TAT protein, successfully used the PTD system to promote neuronal differentiation in murine neural stem cells (20), supporting the fact that the PTD system may be used to drive stem cell differentiation into the desired cell type. However, it is possible that different types of PTD proteins, such as VP22, TAT or Antennapedia, may have different mechanisms of translocation (3). Therefore, since different types of stem cells may behave differently when subjected to the same treatment, it is important to ensure the capacity of VP22 to enter different kinds of stem cells in order to confirm that this protein indeed constitutes a possible tool to be used in stem cell differentiation.

Some studies have observed a biological response of the target cell to the protein introduced by VP22 $(6,8,9,15)$ but none of these addressed stem cells. Preliminary 
results obtained in our laboratory indicate that this VP22 system is also valuable to deliver active proteins into stem cells for in vitro differentiation, since the addition of the VP22.Pax4 fusion protein to a cell differentiation protocol, which leads to insulin producing cells, seems to protect these cells, leading to a higher outcome of insulinproducing clusters and improving the expression of some important genes related to Pax4 (Kossugue PM, Gabanyi I, Lojudice FH, Sogayar MC, unpublished results).

Upon demonstrating the presence of eGFP fluorescence inside two different types of stem cells, namely hDPSCs and mESCs, the present study offers a practical demonstration that VP22 may indeed be a valuable tool to transduce proteins into different types of stem cells. In

\section{References}

1. Phillips MI, Tang YL. Genetic modification of stem cells for transplantation. Adv Drug Deliv Rev 2008; 60: 160-172, doi: 10.1016/j.addr.2007.08.035.

2. Ford KG, Souberbielle BE, Darling D, Farzaneh F. Protein transduction: an alternative to genetic intervention? Gene Ther 2001; 8: 1-4, doi: 10.1038/sj.gt.3301383.

3. Schwarze SR, Hruska KA, Dowdy SF. Protein transduction: unrestricted delivery into all cells? Trends Cell Biol 2000; 10: 290-295, doi: 10.1016/S0962-8924(00)01771-2.

4. Lundberg $M$, Wikstrom $S$, Johansson M. Cell surface adherence and endocytosis of protein transduction domains. Mol Ther 2003; 8: 143-150, doi: 10.1016/S15250016(03)00135-7.

5. Nishi K, Saigo K. Cellular internalization of green fluorescent protein fused with herpes simplex virus protein VP22 via a lipid raft-mediated endocytic pathway independent of caveolae and Rho family GTPases but dependent on dynamin and Arf6. J Biol Chem 2007; 282: 27503-27517, doi: 10.1074/jbc.M703810200.

6. Derer W, Easwaran HP, Leonhardt H, Cardoso MC. A novel approach to induce cell cycle reentry in terminally differentiated muscle cells. FASEB J 2002; 16: 132-133.

7. Ribeiro MM, Klein D, Pileggi A, Molano RD, Fraker C, Ricordi C, et al. Heme oxygenase-1 fused to a TAT peptide transduces and protects pancreatic beta-cells. Biochem Biophys Res Commun 2003; 305: 876-881, doi: 10.1016/ S0006-291X(03)00856-8.

8. Perkins SD, Flick-Smith HC, Garmory HS, Essex-Lopresti AE, Stevenson FK, Phillpotts RJ. Evaluation of the VP22 protein for enhancement of a DNA vaccine against anthrax. Genet Vaccines Ther 2005; 3: 3, doi: 10.1186/1479-0556-3-3.

9. Xiong F, Xiao S, Yu M, Li W, Zheng H, Shang Y, et al. Enhanced effect of microdystrophin gene transfection by HSV-VP22 mediated intercellular protein transport. BMC Neurosci 2007; 8: 50, doi: 10.1186/1471-2202-8-50.

10. Noguchi $\mathrm{H}$, Matsumoto $\mathrm{S}$. Protein transduction technology: a novel therapeutic perspective. Acta Med Okayama 2006; 60: 1-11.

11. Elliott G, O'Hare P. Cytoplasm-to-nucleus translocation of a herpesvirus tegument protein during cell division. J Virol 2000; 74: 2131-2141, doi: 10.1128/JVI.74.5.2131-2141.2000. addition, VP22-mediated protein transduction may be used in stem cell differentiation without disturbing their genome, thus improving the safety of these cells and the reliability of cell therapy protocols.

\section{Acknowledgments}

We are grateful to Dr. Stuart Perkins (Biomedical Sciences Department, Wiltshire, UK) for kindly donating the anti-VP22 antibody. We also acknowledge the excellent technical support of Zizi de Mendonça, Sandra Regina de Souza, Débora Cristina da Costa, Ricardo Krett de Oliveira, and Marluce Mantovani. Research supported by FAPESP, FINEP, CNPq, and BNDES.
12. Martin A, O'Hare P, McLauchlan J, Elliott G. Herpes simplex virus tegument protein VP22 contains overlapping domains for cytoplasmic localization, microtubule interaction, and chromatin binding. J Virol 2002; 76: 4961-4970, doi: 10.1128/JVI.76.10.4961-4970.2002.

13. Brewis N, Phelan A, Webb J, Drew J, Elliott G, O'Hare P. Evaluation of VP22 spread in tissue culture. J Virol 2000; 74: 1051-1056, doi: 10.1128/JVI.74.2.1051-1056.2000.

14. Lemken ML, Wolf $C$, Wybranietz WA, Schmidt U, Smirnow I, Buhring $\mathrm{HJ}$, et al. Evidence for intercellular trafficking of VP22 in living cells. Mol Ther 2007; 15: 310-319, doi: 10.1038/sj.mt.6300013.

15. Saha S, Yoshida S, Ohba K, Matsui K, Matsuda T, Takeshita $F$, et al. A fused gene of nucleoprotein (NP) and herpes simplex virus genes (VP22) induces highly protective immunity against different subtypes of influenza virus. Virology 2006; 354: 48-57, doi: 10.1016/j.virol.2006.04.015.

16. Fang $B, X u$ B, Koch $P$, Roth JA. Intercellular trafficking of VP22-GFP fusion proteins is not observed in cultured mammalian cells. Gene Ther 1998; 5: 1420-1424, doi: 10.1038/sj.gt.3300741.

17. Wang R, Liang J, Jiang H, Qin LJ, Yang HT. Promoterdependent EGFP expression during embryonic stem cell propagation and differentiation. Stem Cells Dev 2008; 17 : 279-289, doi: 10.1089/scd.2007.0084.

18. Lindroos $B$, Mäenpää $K$, Ylikomi $T$, Oja $H$, Suuronen $R$, Miettinen S. Characterisation of human dental stem cells and buccal mucosa fibroblasts. Biochem Biophys Res Commun 2008; 368: 329-335, doi: 10.1016/j.bbrc.2008 01.081.

19. Sukoyan MA, Kerkis AY, Mello MR, Kerkis IE, Visintin JA, Pereira LV. Establishment of new murine embryonic stem cell lines for the generation of mouse models of human genetic diseases. Braz J Med Biol Res 2002; 35: 535-542, doi: 10.1590/S0100-879X2002000500004.

20. Spitere K, Toulouse A, O'Sullivan DB, Sullivan AM. TATPAX6 protein transduction in neural progenitor cells: a novel approach for generation of dopaminergic neurones in vitro. Brain Res 2008; 1208: 25-34, doi: 10.1016/j.brainres. 2008.02.065. 